45 (1) | 2016

En el río del Maíz (Madre de Dios)

Homenaje a Bernard Lelong

\title{
Agir en secret : un regard sur la circulation des usages associés aux souchets des jardins, aux fougères des sous-bois et aux caladions des berges chez les Yanesha (Haute Amazonie péruvienne)
}

Actuar en secreto: una mirada en la circulación de los usos de las juncias de las huertas, de los helechos del bosque y de los caladiones de las orillas entre los yaneshas (Alta Amazonía peruana)

Act in secrecy: a view of the changes in yard sedges, forest ferns and riverbank caladium among Yanesha (Peruvian buffer zone)

\section{Céline Valadeau}

\section{(2) OpenEdition}

Journals

Édition électronique

URL : http://journals.openedition.org/bifea/7913

DOI : 10.4000/bifea.7913

ISSN : 2076-5827

Éditeur

Institut Français d'Études Andines

Édition imprimée

Date de publication : 1 avril 2016

Pagination : 173-192

ISSN : 0303-7495

Référence électronique

Céline Valadeau, «Agir en secret : un regard sur la circulation des usages associés aux souchets des jardins, aux fougères des sous-bois et aux caladions des berges chez les Yanesha (Haute Amazonie péruvienne) », Bulletin de l'Institut français d'études andines [En ligne], 45 (1) | 2016, mis en ligne le 08 avril 2016, consulté le 06 novembre 2020. URL : http://journals.openedition.org/bifea/7913 ; DOI : https://doi.org/10.4000/bifea.7913 de la licence Creative Commons Attribution - Pas d'Utilisation Commerciale - Pas de Modification 4.0 International. 


\title{
Agir en secret : un regard sur la circulation des usages associés aux souchets des jardins, aux fougères des sous-bois et aux caladions des berges chez les Yanesha (Haute Amazonie péruvienne)
}

\author{
Céline Valadeau*
}

\begin{abstract}
Résumé
Ce texte présente un regard sur les possibilités de glissement des usages liés aux plantes d'un corps végétal à un autre. Cette réflexion a été permise par la contextualisation de certains éléments-clés de la botanique et de l'écologie des hautes et des basses terres d'Amazonie. Enrichi par la littérature portant sur certains aspects de la botanique de plusieurs groupes amazoniens, et sur des éléments ethnographiques et historiques choisis, cet article examinera la distribution, le rôle et l'usage des souchets (Cyperaceae), des fougères (Pteridophyta) et des caladions (Araceae) chez les Yanesha, en s'appuyant sur l'histoire de leur migration qui a réparti ce groupe dans des zones d'occupation écologiquement distinctes. Cette démarche propose de repenser la construction des savoirs végétaux à travers les formes de relations que les humains entretiennent avec ces autres espèces du vivant.
\end{abstract}

Mots-clés : cypéracées, aracées, relations interspécifiques, savoirs végétaux, Yanesha, Haute Amazonie

Chercheuse IFEA, Colombia. Ambassade de France en Colombie, Carrera 11\# 93-12, Santafé de Bogotá. E-mail : celine.valadeau@cnrs.fr 


\title{
Actuar en secreto: una mirada en la circulación de los usos de las juncias de las huertas, de los helechos del bosque y de los caladiones de las orillas entre los yaneshas (Alta Amazonía peruana)
}

\section{Resumen}

Este texto presenta una mirada acerca de las posibilidades de cambio de los usos asociados a las plantas de un cuerpo vegetal hacia otro. Esta reflexión fue posible gracias a la contextualización de elementos clave de la botánica y de la ecología de tierras altas y bajas amazónicas. Este artículo mobiliza los aspectos botánicos relevantes y descritos en la literatura con referencia a otros grupos amazónicos, enriquecido con ciertos elementos etnográficos e históricos determinados. En este documento, la distribución, el papel y los usos de las juncias (Cyperaceae), los helechos (Pteridophita) y los caladiones (Araceae) son analizados apoyándose en la historia de la migración del pueblo yanesha, el cual se vio dispersado dentro de espacios ecológicos distintos. Este planteamiento propone repensar la construcción de los saberes vegetales a través de las relaciones que los humanos mantienen con las demás especies vivas.

Palabras clave: Ciperácea, Araceae, relaciones interespecíficas, saberes vegetales, yanesha, Alta Amazonía

\section{Act in secrecy: a view of the changes in yard sedges, forest ferns and riverbank caladium among Yanesha (Peruvian buffer zone)}

\begin{abstract}
This article presents a view of possibilities of shifts in plant usage from one vegetal group to another. These insights were made possible by the contextualization of key botanical and ecological elements of high and low Amazonian lands. Using selected available literature on different botanical aspects as well as ethnographical and historical elements of several Amazonian groups, the distribution, function and use of sedges (Cyperaceae), caladium (Araceae) and ferns (Pteridophytae) by the Yanesha are examined here, based on their migration history that divided this population into distinct ecological zones. The goal of this approach is to reevaluate the construction of plant knowledge based on the relationships that humans maintain with other living species.
\end{abstract}

Key words: Cyperaceae, Araceae, interspecific relationship, plant knowledge, Yanesha, Amazonia Highlands

Dans son ouvrage La rivière du Maïs, Bernard Lelong nous faisait part de son intérêt pour la botanique. Il dévoilait l'existence d'un répertoire dans lequel il avait rassemblé quelque 350 espèces végétales. À l'égard de cet inventaire, il mentionnait « les profondes connaissances botaniques des indiens de la forêt » (Lelong, 1974 : 90). Dans la continuité de cet intérêt porté aux végétaux, le présent article s'interroge 
sur cette même botanique et sur les usages qui lui sont associés dans différentes sociétés amazoniennes et notamment chez les Yanesha1.

La langue yanesha relève de la famille linguistique arawak. Ses locuteurs, chasseurscueilleurs et pratiquant l'essartage, vivent sur les contreforts amazoniens des Andes centrales du Pérou aux abords des fleuves Palcazú, Pachitea, Huancabamba et Cacazú. Les villages sont dispersés dans ces vallées à des altitudes variant de 200 à 1800 mètres. Leur répartition s'explique par différents mouvements migratoires. Les groupes arawak seraient issus du nord-ouest du bassin caribéen, foyer originel des Maipure (Aikhenwald, 1999 : 73). Au cours des siècles, certains auraient migré vers les hautes terres amazoniennes. Aussi, en se basant sur les sièges des évènements structuraux de la cosmologie yanesha, R. Smith démontre que leur territoire est aujourd'hui réduit à une petite zone d'occupation (Smith, 1999 : 59). Leur déplacement s'est fait progressivement, suite aux incursions incas, espagnoles (1553-1576), missionnaires franciscaines (XVlème siècle), militaires (1847), coloniales austro-allemandes (XIXème siècle) et métisses. C'est entre 1847 et 1900 que la majeure partie s'est déplacée vers les basses terres amazoniennes (SantosGranero, 1998 : 131). La population est désormais scindée en deux : aujourd'hui certains se disent teno'marnesha', « les gens de l'amont » et les autres se désignent comme topo'marnesha', « les gens de l'aval ». Malgré cette identification spatiale, tous interagissent avec les constituants d'espaces biotopiques variables qui renferment une ou plusieurs sortes de botaniques yanesha. En gardant à l'esprit I'existence d'une relative porosité entre les catégories végétales, cette botanique est composée d'ensembles complexes qui s'articulent et correspondent sous la forme d'un réseau dense dans lequel les critères organoleptiques et écologiques permettent, de manière circonstanciée, une sorte d'organisation.

Parmi nombre de sociétés amazoniennes, le rapport au végétal s'appréhende sous I'angle de la socialisation (Rama-Leclerc, 2003). L'absorption des plantes transformées en remède permet l'assimilation de qualités indispensables, nécessaires ou souhaitées; le végétal entre intégralement dans le processus de construction de la personne (Valadeau, 2012). La plante ne se dissocie ainsi pas de I'humain puisqu'elle lui est intrinsèquement constitutive. L'acquisition de la qualité d'une plante suppose un ensemble de relations entre les humains et les non-humains dans laquelle la transformation végétale est un processusclé. Cette transformation est au centre du processus opératoire donné par la détermination biotopique, la quête, l'identification de la plante, le chant de cueillette, la cueillette, la transformation par élaboration du remède, le chant thérapeutique, l'absorbation et l'assimilation de la substance végétale, et enfin la diète par évitements alimentaires et comportementaux. La question portera ici sur la compréhension des savoirs végétaux permettant l'identification et l'usage d'une matière végétale d'intérêt.

Les relevés de plantes ont été effectués selon les techniques de la botanique conventionnelle sans écarter pour autant les éléments contextuels comme les 
formes d'énonciation. Le matériel utilisé dans le cadre de cet article est issu d'une recherche ethnographique et d'une étude ethnobotanique réalisées conjointement il y a quelques années. De prime abord, la terminologie végétale possède une grande polynymie ou une véritable polysémie rendant la construction d'une nomenclature délicate. Ce texte est le reflet d'une réflexion qui part d'un constat simple : il existe une réelle variabilité au sein de la botanique yanesha qui est principalement due aux différences de nature de l'environnement avoisinant les villages. Certaines plantes de même dénomination latine sont connues pour leur usage souvent tenu secret dans le bassin amazonien et ses alentours. Ces plantes sont les souchets, les « plantes d'enchantement » et les caladions des berges. Elles possèdent des critères d'usage commun destinant certaines à être utilisées au sein de processus de soin, et d'autres - voire les mêmes — dans des techniques halieutiques ou cynégétiques. L'intérêt porté à ces plantes émane certes de leur singularité en termes d'indication d'usage, mais également de leur reconnaissance majeure dans le monde amazonien. La comparaison entre le savoir botanique des Hautes Terres et celui énoncé par les " gens de l'aval » démontre une variabilité signifiante. Une analyse combinée des critères écologiques de ces espèces, de leurs usages, des mythes et des discours y faisant référence permet de fournir des éléments de réflexion et des hypothèses au sujet des processus de transmission des savoirs de ces plantes discrètes.

\section{LES SOUCHETS DES JARDINS}

Les souchets ou piri-piri en espagnol régional appartiennent à la grande famille botanique des Cypéracées. Ils se répartissent au sein de seulement quelques genres: Carex, Bulbostylis, Cyperus, Eleocharis, Fimbristylis, Scleria, Rynchospora ou encore Torulinium ${ }^{2}$. Ces végétaux de variétés importantes sont souvent cités dans la littérature comme des plantes magiques, médicinales et secrètes (Tournon et al., 1986 : 214 ; Lelong, 1974 : 90). Elles sont vivaces, possèdent l'apparence d'un jonc et se développent en touffes herbacées denses et circulaires (fig. 1). Les souchets sont principalement semés autour des maisons et des refuges des abattis. Ils y sont choyés et, dans une relation presque maternelle, les herbes qui poussent trop près ou tentent de s'enrouler autour de leurs longues feuilles, sont éradiquées. Leur culture est largement pan-amazonienne et la plupart sont domestiqués. Par exemple, chez les Aguaruna du fleuve Mayo certaines variétés sont cultivées près des plants de manioc afin d'aider la maturation des tubercules (Brown \& Van Bolt, 1980 : 182), certaines autres sont identifiées comme sauvages (Jauregui et al., 2011 : 748 ; Kohn, 2002 :178 ; Tournon et al., 1986 : 218).

Leurs usages sont tenus secrets par leurs propriétaires, même si leurs rhizomes sont souvent objet d'échange. Leurs similitudes morphologiques ne permettent pas de les différencier et seule la personne qui les soigne les connaît individuellement. En

2 C'est principalement le genre Cyperus qui est retenu. Beaucoup de dénominations peuvent être associées à ce seul genre. 


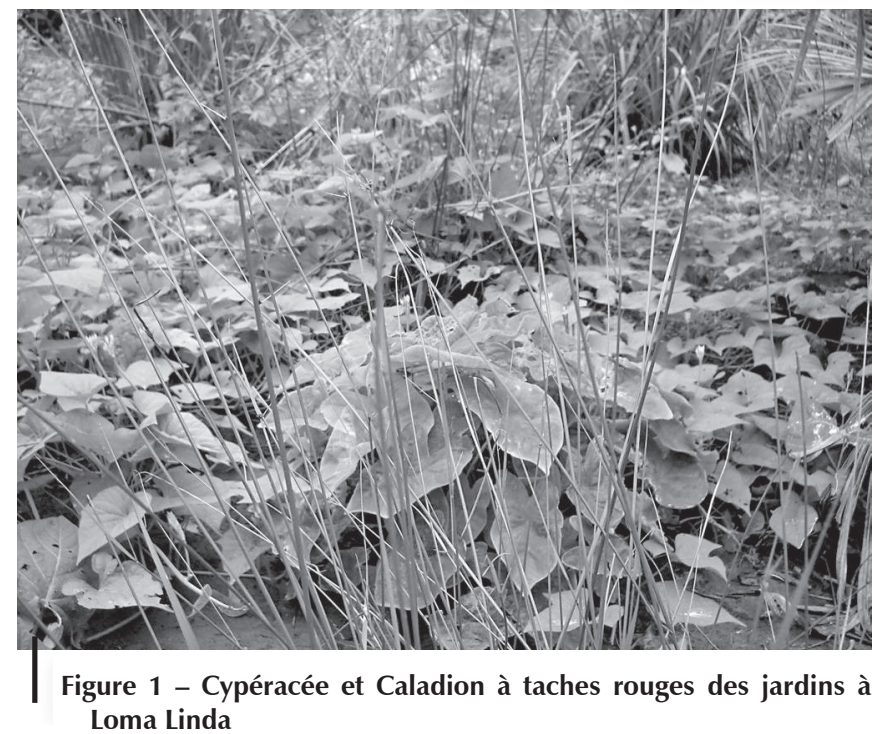

(C) Céline Valadeau

Amazonie, leurs usages sont relativement homogènes. La plupart de ces plantes sont utilisées comme des charmes de chasse ou de pêche chez les Shipibo-Konibo du Haut Ucayali (Tournon et al., 1986 : 222 ; Belaunde, 2012 : 127), les Ashaninka du Pichis (Rivas Ruiz, 2004 : 174), les Matsigenka du Haut Manú (Shepard, 1999 : 104), les Amahuaca de Purús (Carneiro, 1970 : 339) ou encore les Mai Huna du fleuve Napo (Bellier, 1986 : 468). Les techniques de préparation décrites sont relativement semblables : en général, les tubercules sont mâchés ou réduits en poudre et mélangés à une préparation de feuilles écrasées avec un peu d'eau ou de salive formant une pâte avec laquelle sont frottés les outils de prédation. D’autres sont utilisés comme remède vétérinaire et sont destinés aux chiens de chasse. Chez les Shuar d'Équateur, la préparation du rhizome écrasé dans un peu de salive est instillée dans I'œil (Bennett, 1989 : 59). Les Chayahuita du sud-ouest du Loreto leur en administrent par voie orale (Ochoa-Gilonne, 2007 : 180).

Ces plantes peuvent aussi participer à rétablir un état de santé altéré. Les usages recueillis dans la littérature sont très nombreux. Sans s'adonner à l'établissement d'un répertoire exhaustif, en voici quelques-uns33. Les propriétés fébrifuges sont connues des Wayampi du Haut-Oyapock (Grenand, 1980), chez les Yanomami du Venezuela (Milliken \& Albert, 1996 : 17) ou encore au sein du groupe frontalier yagua (Chaumeil, 1993 : 284). Elles facilitent l'accouchement et possèdent une action contraceptive chez les Jivaro, Huitoto, Yagua et Shipibo-Konibo (Maxwell, 1970 : 106). Des notes sur leur action antidiarrhéique ont été recueillies auprès

3 Ne sont mentionnés ici que certains des usages indiqués par les auteurs. En effet, plusieurs usages sont en général répertoriés. 
des Aguaruna ou des Shipibo chez qui le pouvoir hémostatique a été mentionné (Tournon et al., 1986 : 222). Les souchets sont aussi d'excellents fortifiants pour les nouveaux-nés yagua (Chaumeil, 1993 : 239) et ashaninka du fleuve Quimiriki (Luziatelli et al., 2010 : 7). Par ailleurs, les Shuar d'Équateur les utilisent contre les morsures de serpent (Bennett, 1990 : 57). Là, les procédés de préparation et d'administration sont aussi sensiblement comparables : la plupart du temps le rhizome est mâché, bu en infusion, instillé dans les yeux ou utilisé sous forme de vapeur. Sa combinaison avec le tabac (Nicotiana tabacum), I'ayahuasca (Banisteriopsis caapi) (Schultes \& Hofman, 1979 : 124) ou encore le sanango (Tabernaemontana sananho) a également été décrite (Kohn, 2002 : 178). Dans certaines préparations chayahuita, ils sont mélangés à du huito (Genipa americana) afin de lever l'effet d'un sort jeté sur un enfant (Odonne et al., 2013 : 151).

Par ailleurs, les Cypéracées seraient puissantes au point d'avoir une action météorologique. Ainsi, chez les Runa Quichua, la fumée d'Eleocharis sp. animerait le vent et éloignerait les nuages tandis qu'une eau infusée répandue attirerait l'orage (Kohn, 2002 : 178). Ces interactions écologiques ont été constatées chez les Sharanahua du fleuve Purus qui dans le mythe «le maître avaricieux » mentionnent que certains piri-piri auraient le don de faire pleuvoir (Del Aguila \& Déléage, 2010 : 40).

L'origine des souchets a parfois été décrite dans la littérature. Les Cyperus cynégétiques aguaruna auraient été obtenus grâce à la chasse d'un vautour : le cadavre aurait été brûlé et les piri-piri se seraient épanouis de ses cendres. Leurs usages se dévoilent par la suite. Par exemple, si un chasseur porteur d'un rhizome de carex croise un boa, cela signifie que ce souchet attire spécifiquement ces serpents. Dès lors, il sera choisi de le cultiver ou non (Brown, 1984 : 553). Les Shipibo-Konibo, les Chayahuita les Quechua du Pastaza connaissent un mythe semblable. Les souchets seraient apparus à l'endroit où soit les cendres soit les dépouilles de petits animaux furent enfouies (Tournon et al., $1986: 217$; OchoaGilonne, 2007 : 187 ; Gutierrez Choquevilqua, 2013 : 38).

Chez les Yanesha, les souchets se nomment génériquement epe ${ }^{\prime 4}$. Les différents mythes relatant l'origine des souchets sont construits de manière semblable. Ils racontent comment un inconnu peut empêcher une action de pêche, de chasse ou de combat et comment à l'aide d'une supercherie, le coupable est démasqué et contraint au don. Le mythe de l'apparition des souchets de chasse a été décrit ainsi :

On dit qu'auparavant vivait un chaman qui était un excellent chasseur. En forêt sa chance était incalculable, il en rapportait tapirs, daguets et bien d'autres animaux. Quand un jour, un fait étrange se produisit. Arrivant sur les lieux où les animaux s'étaient supposément effondrés, il ne trouvait plus rien.

4 Chaque souchet porte aussi un nom spécifique qui se caractérise par la présence du suffixe -phar, uniquement employé pour cette catégorie de plantes. 
Poramosa', le Père de tous les oiseaux, possédait des souchets comme auxiliaires. Chaque fois qu'un animal était abattu, ces souchets lui indiquaient la direction dans laquelle il se trouvait en pliant leurs feuilles.

Alors mécontent, le chasseur partit voir son ami Pabhchar et lui conta ses mésaventures. Ce dernier lui confia que c'était Poramasa' qui était à I'origine de son désarroi et lui suggéra une supercherie : " II faut organiser un piège, il viendra ».

Le jour suivant, le chasseur tua à bout portant plusieurs tapirs et eut l'idée de se cacher dans le ventre de l'un d'eux. Aussitôt, des carnassiers arrivèrent et encerclèrent les proies ensanglantées attendant ainsi la venue de Poramasa' qui seul possédait le couteau permettant de découper le corps pour en sortir les tripes et se nourrir. Pendant ce temps, les oiseaux ventilaient avec leurs ailes afin de préparer la viande à être consommée.

Une fois sur les lieux, Poramasa' trancha le ventre du tapir dans lequel se cachait le chasseur. L'homme bondit et s'empara du couteau. II fit quelques pas en arrière en disant : «Si tu veux ta lame, tu dois laisser mes proies. » Surpris, Poramasa' répondit : « Laisse-moi rentrer chez moi, je vais te l'échanger contre quelque chose qui te sera utile ». II s'en alla et à son retour, il dit au chasseur : «Tiens, en échange, voici des herbes epe'. Elles attirent les proies. Sème-les chez toi. Explique ceci à tes proches. Protège-les bien. » C'est en enseignant I'usage des souchets de chasse que Poramasa' reprit son outil.

Des narrations similaires existent dans d'autres mythologies arawak comme celles des Ashaninka (Rivas Ruiz, 2004 : 174). Les usages de ces plantes ont une correspondance directe avec leur mythe de référence. En règle générale, les epe' apportés par Yato' Yos, la Divinité Suprême, renvoient soit à des qualités individuelles valorisées dans la vie sociale, soit à des usages faisant référence aux processus de construction de la personne et plus précisément du yecamkëmh, principe de vitalité de la personne. Ce dernier est agrémenté avec des vitalités de plantes dont l'usage de chacune est défini par la nature même de sa propre vitalité (Valadeau, 2012 : 43). Par exemple, morreñtsophar (morreñets : " chanter»; Cyperus sp.) fut offert par Yato' Yos afin que chaque personne puisse acquérir des facultés d'apprentissage importantes, facilitant la mémorisation des chansons et des mythes. Dans cette même logique, cacphar (cac : «poisson »; Cyperus sp.) est un don du martin pêcheur Shemoc Huarena' qui apporta les epe' de pêche ; I'aigle harpie Rrera donna ceux utiles à la chasse aux oiseaux ; pochenrrophar (cheneñets : « rompre un morceau de bois »; Cyperus sp.) fut un présent du guerrier Ransana' afin d'être valeureux et de pouvoir rediriger les armes envoyées dans sa direction, et les cypéracées de Ransana' permettent d'attirer les armes et les éléments malintentionnés vers soi afin de les désactiver avant qu'ils n'arrivent à destination ; taneñetsophar (taneñets : « filer ») a été laissé par l'araignée Arancmana' afin de dompter le coton, pour qu'il soit docile et s'agence facilement sur la canule. La dernière caractéristique de ces plantes réside dans leur interaction avec d'autres espèces et notamment avec certains colibris. Ceux dont le bec est 
de morphologie courbée se nomment aseñac et visitent les epe' des jardins. Les Yanesha considèrent leurs venues comme prémonitoires : elles annoncent des personnes malintentionnées, cherchant à procéder à de la sorcellerie. L'interaction entre ces colibris insectivores et les souchets se fait par l'intermédiaire de petites araignées qui tissent des toiles semblables à des petites boules de coton culminant sur les inflorescences hautes des cypéracées.

En ce qui concerne les techniques de préparation, à l'instar d'autres sociétés amazoniennes, ce sont les parties tubérisées du rhizome qui sont utilisées. Le procédé varie en fonction de l'usage recherché. Les souchets permettant d'avoir des qualités particulières sont en général écrasés et ingérés avec un peu d'eau. Les rhizomes de piri-piri de charme, de chasse ou de pêche sont réduits en poudre qui peut être humectée avec un broyat de leurs propres feuilles. La masse obtenue est frottée entre les mains, sur les bras et sur les armes. Le procédé est le même avec les outils de tissage. Lors de la chasse ou de la pêche, un tubercule est souvent mâché et soufflé autour de soi dans le but d'aveugler les entités malignes environnantes. Dans le cas d'un épisode fiévreux ou diarrhéique grave ou afin d'accélérer un accouchement, les tubercules sont mâchés et soufflés par le végétaliste sur le corps. Pour terminer, certains souchets, comme yataphar (Cyperus laxus), peuvent aussi être ajoutés aux décoctions de tabac que confectionnent les chamans.

L'ensemble des souchets yanesha semble se scinder en deux groupes se distinguant I'un de l'autre par leurs usages. Le premier est constitué des souchets maîtres c'est-à-dire des plantes de Yato' Yos utiles à la construction de la personne et le second est formé par l'ensemble des Cypéracées de charme. Cette dernière catégorie regroupe les plantes qui possèdent un pouvoir attractif : celui de diriger les poissons et les animaux de proie vers soi afin de les abattre, comme celui d'attirer les armes dans le but de les annihiler ou celui de rendre docile le coton afin de le contraindre par le tissage.

\section{LES PUSANGA ET LES FOUGÈRES DES SOUS-BOIS}

Les pusanga sont des plantes d'une odeur délicieuse qui sont parfois identifiées comme des piri-piri (Tournon \& Silva, 1988 : 171 ; Jauregui et al., 2011 : 748). Dans le cas contraire, la nature botanique reste souvent imprécise. Ces plantes entrent dans la composition de philtres d'amour : " "pusangas": charms used to manipulate the perceptions, intentions and behaviors of others » (Alexiades, 1999 : 267). Elles s'utilisent " comme parfum pour faire "perdre la tête" aux femmes » chez les Sharanahua (Déléage, 2005 : 257). Pour les Ese Eja, leur senteur sucrée est associée au charme sexuel puisqu'elle attire et séduit (Alexiades, 1999 : 271). La frontière entre l'attraction et la séduction forcée est mince (Carid Naveira \& Pérez Gil, 2002 : 259). Cela requiert différentes méthodes pouvant aller de la simple application du parfum ou de l'apposition d'une mince quantité de préparation sur les vêtements ou les cheveux de la personne convoitée, à l'enfouissement d'un petit sachet contenant la pusanga préparée aux abords de la maison où vit l'être désiré. Cette dernière technique est perçue comme une agression chamanique chez les 
Yaminahua de la région d'Atalaya du Pérou (Carid Naveira \& Pérez Gil, 2002 : 259). Chez les Matsigenka, les agressions perpétuées à l'aide de ces « potions d'amour » sont liées à des actes de sorcellerie piro ou ashaninka qui provoquent des perturbations sociales (Shepard, 1999 : 155). Enfin, d'autres pusanga ont des usages médicinaux comme chez les Ese Eja où la pusanga de gallinazo (Mansoa Alliacea) et la pusanga de maquisapa (Drymonia semicordata) sont respectivement utilisées pour soigner les dermatites et les céphalées (Alexiades, 1999 : 395, 399).

Pour leur part, les Yanesha traduisent le terme générique espagnol pusanga par celui de puerets (pueretsoteñets : " faire un traitement efficace », pueseñets : « oublier »). Les puerets sont au nombre de cinq : epo'puer (puerreñets : « revenir »), onen (oneñets : " être sur le point de pleurer »), yahue' (yahueñets : « pleurer »), yayoñ (yoñ : « tête ») et yerpuen (yerpueñets : " se souvenir »). Ces plantes sont des ptéridophytes. Elles poussent en forêt dense et sont difficiles à trouver puisque, de coutume, " elles se cachent lorsqu'on les cherche ». Peu de gens savent - ou désirent - indiquer les lieux où elles seraient susceptibles de s'épanouir. De par son importante morphologie arborescente, yahue' (Blechnum peruvianum) est la première espèce qui a été identifiée. Les quatre autres fougères Epo'puer (Serpocaulon sp.), onen (Serpocaulon loriceum), yayoñ (Polypodium laetum) et yerpuen (Solanopteris bifrons) sont de petite taille. Toutes ces ptéridophytes développent une odeur agréable lorsque leurs rhizomes sont écrasés. Par ailleurs, leurs feuilles ont une croissance caractéristique. Par exemple, les feuilles de yahue' passent d'un vert émeraude à un rouge grenat au fur et à mesure de leur maturation. Epo'puer, onen, yayoñ et yerpuen sont épiphytes et leurs sores (fructifications des fougères) passent d'une couleur jaune à bronze. Dans le groupe arawak voisin des Ashéninka, des fougères de séduction telle la plante du singe hurleur et de la séduction sans frein ont également été répertoriées (Tectaria incisa, Stigmatopteris sp. et/ou Pteris sp.) (Lenaerts, 2002 : 198).

Les caractéristiques organoleptiques de ces plantes se retrouvent dans le mythe d'origine des puerets. Ces plantes étaient connues comme cinq très belles jeunes filles aux vêtements colorés et aux parfums subtils. Leur compassion envers les Yanesha était immense. Mais si leurs motivations étaient pures et leurs actes protecteurs, elles usèrent néanmoins de leurs charmes afin de forcer Huana à prêter attention à leur cause :

Huana était un homme qui avait le pouvoir de faire tomber la pluie là où il regardait 5 . C'est pour cela qu'il se concentrait sur la fabrication de couronnes et de flèches. Malgré cela, il pleuvait sans discontinuer.

On dit que Yerpuen, Onen, Yayoñ, Yahue et E'popuer étaient de belles et puissantes jeunes filles très agréablement parfumées vêtues de tuniques, de bracelets et de sacs multicolores ornés de motifs. Un jour de forte pluie, elles s'en allèrent voir Huana. [...] Lorsqu'elles le saluèrent, il n'en fit cas. II resta concentré sur son ouvrage et feignit de ne pas les entendre. Les jeunes

5 Il est possible de noter ici le parallèle existant entre le pouvoir des piri-piri sharanahua de faire pleuvoir et le désir des puerets d'arrêter la pluie. 
filles le supplièrent d'arrêter la pluie. Elles insistèrent : « Huana, puissant Huana, les rivières sont pleines, le manioc se noie et pourrit dans les terres détrempées. S'il te plait, aide-nous !» [...]

Il ne les écouta pas et elles se détournèrent, lorsqu'elles firent demi-tour leur parfum inonda Huana qui les regarda du coin de l'œil. Quelques instants plus tard, Huana pris de remords se mit à courir pour les rejoindre. «Elles ne doivent pas être loin !» se disait-il. [...] Mais, elles ne l'avaient pas attendu et cheminaient en direction de Huanuco. Huana chercha et cria leurs noms de tous les côtés : «Les filles, les filles ? Où êtes-vous parties? Parlons maintenant, je vais arrêter la pluie! Répondez-moi ! seul et fatigué, Huana se lassa.

Lorsque les Pères suprêmes se dirigèrent vers les cieux, les jeunes filles prirent la forme de plantes et gardèrent leurs noms. Leurs nouvelles formes végétales donnèrent la possibilité aux humains d'acquérir leurs qualités. C'est ainsi que yayoñ donna à une personne la possibilité de paraître très belle aux yeux d'une autre de sexe opposé ; epópuer permet de paraître important à une personne initialement indifférente, mais qui cherchera ensuite à la retrouver ; yerpuen permet que quelqu'un se souvienne incessamment de soi ; onen et yahue' convoitent le fait qu'un être désiré pleure ou soit toujours sur le point de pleurer en son absence.

Une ambigüité entre maîtrise d'autrui par usage de charmes et affirmation de soi caractérise l'usage qui est fait de ces plantes. II est reconnu qu'au sein de la société, certaines personnes ne se satisfont pas de l'idéal proposé. Elles cherchent à développer des qualités personnelles particulières qui vont faire d'elles des êtres à part. Cette démarche vise à acquérir une maîtrise spécifique tel un don musical hors normes ou toute autre activité dont la réalisation est jugée particulièrement chanceuse. C'est dans ce but que les puerets étaient initialement connues et utilisées. Selon les anciens, les puerets étaient auparavant des végétaux dont I'usage était strict et qui permettaient de se changer soi-même. Leur maniement était exclusivement effectué dans un cadre humble et modeste, dépourvu de toute trace de vanité, d'orgueil ou de recherche de possession au risque de se voir devenir fou ou de tomber gravement malade. En effet, le danger de devenir une personne hors normes, manipulatrice, orgueilleuse et vaniteuse est toujours présent et se sanctionne invariablement par la folie. Dans ce contexte, l'action ambiguë des puerets procure un sentiment qui, selon les motivations, peut s'apparenter à celui d'un bien être personnel, pouvant facilement basculer dans une sorte d'hypertrophie de l'ego, nuisible à la fois pour la personne comme pour l'ensemble de la communauté. II s'agit de trouver un équilibre entre des aspirations égoïstes et un réel désir de s'améliorer afin d'optimiser ses relations avec les autres. Aujourd'hui, les puerets sont utilisées dans un but social, pour faire en sorte d'établir de bonnes relations. En effet, la personne idéale est non seulement productive, mais elle entretient aussi des rapports harmonieux avec l'ensemble du groupe, dont son conjoint. Les personnes mariées sont décrites comme partageant une partie de leurs vitalités. L'imprégnation mutuelle est mise 
en pratique du fait de leur présence rapprochée. Cela explique pourquoi certains puerets sont utilisées tout au long de la vie conjugale, non pas dans le but de rendre I'autre en adéquation avec soi, mais pour modifier son propre yecamkëmh afin qu'il s'harmonise. Lorsque les yecamkëmh conjoints se partagent, apparaissent les sentiments d'attachement tels que l'amour, morrenteñets ou l'amitié, yemteñets. Les puerets sont donc des plantes qui permettent d'accéder à l'intimité d'une personne, dans une dynamique d'échange, de partage et de maîtrise émotionnelle.

Actuellement, ces formes d'usage des puerets ne sont pas les plus largement connues ni mentionnées. Ces fougères sont davantage décrites comme permettant de modifier les sentiments et les attraits des personnes de sexe opposé dans le but de transformer les relations affectives. Les manipulations relationnelles qu'entraîne l'usage de ces végétaux passent pour dangereuses. D'un point de vue fonctionnel, les puerets modifient le yecamkëmh de la personne visée par apposition à son insu d'une petite quantité de potion sur ses vêtements, c'est-à-dire en lui agrégeant les vitalités du puerets sélectionné. Celle-ci, initialement indifférente, va soudain devenir déraisonnablement intéressée par cet individu, qui le plus souvent ne répond pas aux critères du partenaire idéal. De fait et dans ce contexte, le terme puerets a adopté spontanément la dénomination espagnol pusanga. Les cas de manipulation émotionnelle dus aux puerets sont des faits de sorcellerie. Des personnes malintentionnées se complaisent parfois à déposer sur d'autres une potion composée d'une poudre fine de sores et de rhizomes de fougères mélangés à une décoction réduite de roucou (Bixa orellana) amalgamée de graisse de poule6. Dès lors, ces personnes n'arrivent plus à suivre les règles sociales. Elles vont quitter leur domicile, courant dans n'importe quelle direction dans le but de rejoindre la personne qu'elles pleurent sans cesse et qui leur a administré le puerets à leur insu. Or, l'identité de la personne malveillante est souvent inconnue. Leurs victimes deviennent « folles », prises « d'attaques répétées et chroniques » telles des crises épileptiques. Les seuls remèdes permettant de pallier aux effets des puerets sont les «plantes de l'oubli », puesen. À chaque puerets correspond un puesen spécifique. Si les puerets modifient les yecamkëmh par addition de leur propre vitalité, leur soustraction est alors possible grâce à l'usage de puesen seulement si le puerets est connu, ce qui est rarement le cas.

Pour conclure sur l'usage de ces fougères, les puerets ne sont pas des objets d'échange, contrairement aux souchets. Sylvestres, non cultivées et peu abondantes, ce sont leurs usages qui semblent se distribuer. Toutefois, il existe des «puerets non yanesha » tels a'coshañpo'se' (lit. dauphin rose de l'Amazone) qui sert à attirer l'attention d'une femme, casantaperes (lit. espèce de petit oiseau jaune) pour être performant et mettre en valeur la quantité de travail effectué ; huankëñets (huankëñets, lit. se nouer) qui permet de lier deux personnes ; meshe'llopar (meshe'll, lit. espèce de petit oiseau) qui a le don d'attirer une femme en la détournant de l'attention qu'elle a sur ses enfants. Ces puerets ont été cités

6 Le roucou aurait la capacité de conserver le pouvoir de la plante. 
par les Yanesha comme des plantes que les Ashaninka et les Shipibo-Konibo leur ont apprises lors de marches communes en forêt.

Les puerets, en tant qu'espèces permettant des modifications profondes du yecamkëmh des personnes, sont donc des plantes qui jouent un rôle important dans le domaine de la fabrication des relations affectives. Elles peuvent être perçues comme des plantes capables de renforcer un critère jugé idéal. Selon leur emploi, les modifications induites par les puerets visent à se transformer soi-même ou à bouleverser autrui, afin d'être en plein accord avec ses propres désirs. Il existe deux formes d'usage permettant les transformations individuelles : I'une réalisée dans un but social — référant à leurs anciens usages — et l'autre accomplie afin de renforcer l'unité conjugale, un usage qui semble être plus contemporain.

\section{LES ARACÉES DES RIVES}

Les aracées cultivées sont des plantes peu décrites. Pour la plupart, elles appartiennent aux genres Caladium, Xanthosoma, Alocasia voire Colocasia dont

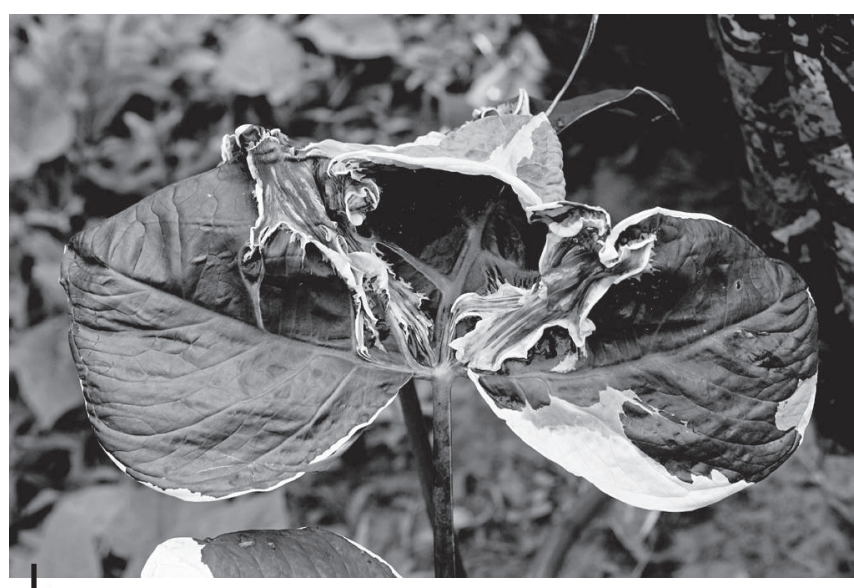

Figure 2 - Feuilles de Xanthosoma à taches blanches de Nueva Esperanza

(C) Céline Valadeau la diversité est importante à la périphérie du bassin amazonien (Madison, 1981 : 342) (fig. 2). Leur usage n'est pas un phénomène récent puisque Cunha les désignait déjà sous le terme mangará et en signalait l'existence au XVI siècle. Plus tard, Grégorio associera ce terme aux Caladium (Grenand \& Fleury, 2012 : 15). Ces plantes sont herbacées, leur racine tubéreuse et leurs feuilles cordiformes dont les couleurs varient d'un vert ponctué de blanc au pourpre. Cette coloration variable définit un nombre élevé de cultivars parfois nommés palettes de peintre. D'un point de vue écologique, elles couvrent les sols ensoleillés comme les clairières, les berges des rivières et les bords des chemins. Ces aracées sont domestiquées dans de nombreuses sociétés d'Amazonie où elles poussent dans les jardins, aux abords des maisons et dans les abattis. Caladium bicolor et Xanthosoma sp. sont, par exemple, cultivés par les Aguaruna près des plants de manioc (Katz et al., 2012 : 288).

De nombreux écrits mentionnent leur présence en tant que composante du paysage comme chez les Runa Quichua d'Équateur (Kohn, 2002 : 412), chez les Jodï du Venezuela (Zent \& Zent, 2012 : 327) ou encore chez les Wayana de Guyane (Chapuis, 1998 : 1186). Il existe peu de littérature décrivant leurs usages, même si quelques textes apportent des témoignages intéressants sur leurs utilisations. Par 
exemple, les Wayampi frottent sur leur corps les tubercules de Caladium bicolor contre les esprits, en cas de «folie», pour l'ensemble des maladies de l'enfance ou en tant que contrepoison (Folliard, 2008 : 241). Les Palikur les utilisent en poudre frottée sur le visage pour faire disparaître les masques de grossesse ou certaines taches de la peau (Folliard, $2008: 242$ ). Un répertoire dense sur l'usage de ces plantes dans le bassin des Guyanes a récemment été publié et apporte énormément à la connaissance de leur utilisation (Van Andel et al., 2015).

Les Aracées des Wayampi sont des charmes d'amour. Le tubercule écrasé de I'arum de la grenouille (Xanthosoma conspurcatum) mélangé à du roucou constitue un philtre d'amour lorsqu'il est appliqué à l'aide d'un petit bâton sur la personne convoitée (Folliard, 2008 : 241). Non loin, en Guyane britannique, les Akawaio I'utilisent comme philtre d'amour ou d'habileté (Van Andel et al., 2015 : 4). Les femmes yanomamë du Brésil procèdent à des magies amoureuses avec des poudres de Caladium sp. (Albert, 1985 : 261).

Par ailleurs, ces plantes ont des usages cynégétiques; par exemple les Piaroa ont recours aux caladions des jardins lors de rituels mineurs de chasse (Heckler, 2004 : 223). Sur le plateau des Guyanes, ces plantes sont principalement utilisées en tant que charme de chasse (Van Andel et al., 2015 : 4). Chez les Wayampi, le tubercule est emporté dans une musette et les feuilles préparées servent à oindre le visage et les poignets avant la chasse. Chez les Tupi-Guarani de Guyane, un rite cynégétique consiste à frotter le tubercule de l'effraie-serpent (Caladium schomburgkii) sur les jambes lors des chasses afin que les serpents aient peur de s'approcher (Grenand, 2001 : 30)7. Tout comme les souchets, à chaque animal correspond un tubercule spécifique : taiteku pola (Caladium bicolor) pour les pécaris à collier et tapi'i pola (Xanthosoma sp.) pour les tapirs. De la même manière, les arums de la loutre sont utilisés comme charme de pêche (Folliard, 2008 : 241), usage aussi retrouvé au Pérou chez les Shipibo (Tournon \& Silva, 1988 : 173) et chez les Shawi où ces plantes de charmes adoptent aussi le nom espagnol pusanga (Odonne et al., 2014 : 45).

À l'instar des puerets, ces aracées sont ambivalentes. En effet, des usages maléfiques ont été mentionnés : les Palikur jettent une pincée de tubercule râpé dans la bière de manioc afin de l'envenimer (Folliard, 2008 : 242). Chez les Yanomani, yoboro est un Xanthosoma cultivé et utilisé selon la méthode de prise d'empreinte ou frotté sur le hamac de la personne choisie afin que son corps entre en putréfaction (Albert, 1985 : 269).

Dans le piémont central du Pérou, les aracées sont utilisées dans les villages yanesha localisés à une altitude inférieure à $900 \mathrm{~m}$. Les Caladium ne poussant pas à des altitudes bien supérieures, la raison est simplement écologique. Les aracées utilisées sont domestiquées et semées autour des maisons. Á la manière des

7 Chez les Yanesha un usage semblable est décrit pour la tige d'une autre aracée (orroshe'sh : Dracontium spp.) dont l'aspect ressemble aussi à celui de la peau d'un serpent (Valadeau, 2012 : 108). Cette caractéristique morphologique est aussi notée chez les Ashéninka (Lenaerts, 2002 : 185). 
souchets, leurs usages sont gardés secrets et leurs organes souterrains deviennent parfois un objet d'échange.

II n'a par ailleurs pas été répertorié de mythe relatant leur apparition, seules des bribes ont été évoquées. De manière assez uniforme, les discours se construisent autour de l'idée commune selon laquelle les aracées sa'poque' auraient poussé sur les rives à partir des cendres enfouies de différentes proies. Une anecdote rapportée par une femme ayant quitté le village en direction de Villa Rica raconte comment elle a tué le boa qui lui barrait la route. Elle n'aurait pas dû l'abattre à coup de bâton s'il ne l'avait pas fixé du regard quelques instants. Des semaines plus tard, elle rapporta un caladion du lieu de leur rencontre et le planta dans son jardin8.

Les usages attribués aux aracées sont divers. Certains sont semblables à ceux des puerets puisque les tubercules sont destinés à permettre l'union des personnes, leur attachement irrévocable ainsi que la fertilité des femmes. D’autres sont décrits tels des charmes utilisés dans les rituels mineurs halieutiques ou cynégétiques.

Ces plantes sont communément et principalement connues des femmes qui les choient en les arrosant périodiquement avec un peu de bière de manioc ${ }^{9}$. Les tubercules écrasés de sena'm (Xanthosoma sp. ou Maranta sp., une Marantaceae aux longues feuilles ovales bigarrées de rayures bleutées) sont cuits jusqu'à l'obtention d'une préparation crémeuse qui est refroidie avant d'être filtrée à l'aide d'un linge (fig. 3). Le résidu emprisonné dans le tissu est étalé dans un récipient qui est exposé au soleil. Une fois sec, une fine poudre est recueillie en raclant doucement le support sur lequel elle s'est agglomérée. Elle est rassemblée dans un petit tube de bambou afin d'y être conservée. Cette poudre est utilisée de différentes manières dans le cadre d'un rituel durant plusieurs jours. Afin d'attacher les vitalités des personnes, le couple s'en frictionne le corps avant de dormir. Une petite quantité est aussi déposée discrètement à l'entrée du vagin avant l'acte

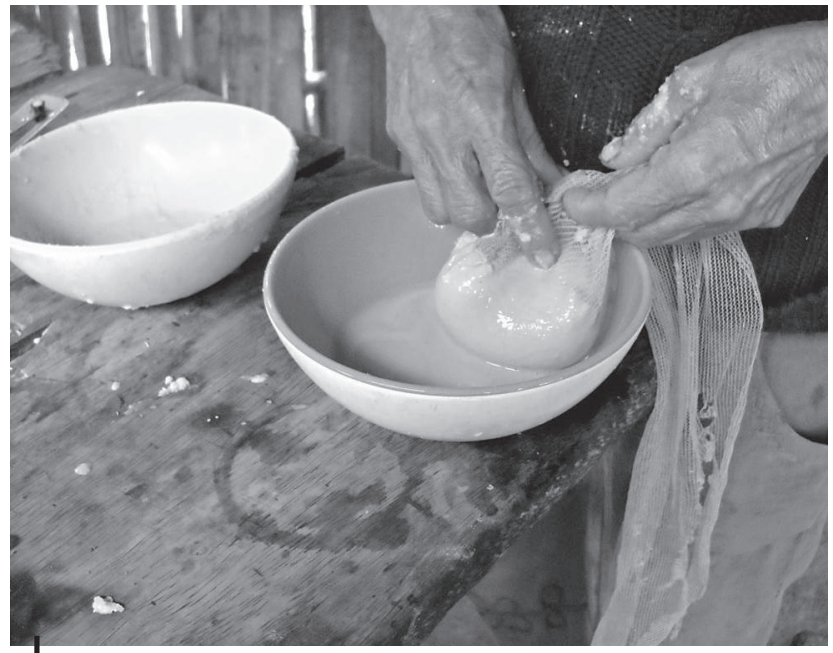

Figure 3 - Filtrage de la préparation d'Araceae dans un linge (C) Céline Valadeau

8 Selon cette femme, après avoir abattu le boa, elle s'est enfui. Elle est rentrée chez elle pour demander de l'aide afin de transporter la dépouille de l'animal pour en soustraire la peau et la vendre. Le boa n'était plus là lorsqu'ils arrivèrent quelques heures plus tard (anecdote recueillie à Azulis en 2009).

9 Le fait d'être une femme m'a permis de pouvoir partager de nombreux secrets féminins. Par contre, le savoir des hommes en la matière a été plus difficilement accessible. 
sexuel que précède les frictions corporelles. Le rhizome râpé à l'aide d'une cuillère afin d'obtenir une masse pâteuse blanchâtre est mélangé à un mets ou à un breuvage afin que I'homme l'ingère à son insu. Ces dernières techniques évitent aux conjoints de se contredire et rendent leur éloignement impossible. Une indication semblable a été notée chez les caboclos du fleuve Madeira au Brésil : his wife will follow him around like a puppy, responding to his every command! (Kawa, $2011:$ 128).

Par ailleurs, d'autres Caladium bicolor sont des charmes de chasse. Ils sont utilisés en poudre et frottés sur les outils de prédation pour attirer le gibier ou le poisson. De la même manière, quelques pincées de poudre mélangées à un broyat de feuilles forment une masse qui est frictionnée entre les mains avant d'aller pêcher des crevettes que l'on peut alors simplement capturer en plongeant les mains dans l'eau.

Certains autres cultivars de feuilles à prédominance rouge sont décrits comme des «plantes de jeu ». Nommées jeretso'pan (jeretso' lit. Feu du diable), elles attirent les choyeshe'mats, ombres des défunts, et des entités malfaisantes comme Jo', lorsqu'elles rodent autour des maisons. Occupés à jouer, ces malfaisants ne pensent plus à autre chose. Ces aracées ont donc un pouvoir d'attraction envers les humains, les animaux et les entités du monde non visible.

Enfin, d'autres usages ont été relevés. Les feuilles ponctuées de blanc de Cheyortsopan (Xanthosoma sp.) peuvent stimuler la fertilité des femmes.

Pour terminer, il est envisageable de distinguer deux groupes distincts d'aracées définis en fonction de leurs usages. Certains prolongent les principes de fabrication relationnelle — rencontrée également chez les puerets d'union - et d'autres ont des visées prédatrices. Il est donc possible de distinguer respectivement des aracées d'union et des aracées de charme. Dans un sens, ce clivage se retrouve au sein des piri-piri qui possèdent d'une part le pouvoir d'agir sur la composante principale de la personne et d'autre part celui d'attirer les proies et de leurrer d'autres espèces.

\section{INDICATIONS D'USAGE ET AUTRES FORMES VÉGÉTALES}

Le point commun de la plupart de ces formes végétales réside dans le pouvoir attractif qu'elles renferment. Ce dernier, constitué de la nature même de la vitalité végétale, possède la caractéristique majeure d'avoir une odeur délicate. Ces plantes sont toutes odoriférantes. Les rhizomes des souchets, ceux des fougères et la fleur des caladions développent des arômes caractéristiques. Lors de l'épanouissement de leur fleur, une odeur fruitée attirant les coléoptères polinisateurs se dégage pendant deux nuits consécutives. Tout comme ces insectes aux élytres colorés, le gibier et le poisson sont attirés vers qui s'associera à leur fragrance.

Dans le cas des puerets comme des aracées d'union, les humains peuvent en user pour attirer et pour séduire avec une certaine ambivalence puisqu'elles peuvent aussi bien servir à porter préjudice à autrui par des actes de sorcellerie 
qu'à amplifier I'union de deux êtres. Les facultés attractives sont les propriétés principales des souchets comme des aracées de charme ${ }^{10}$.

Dans certains cas, d'autres capacités individuelles peuvent être acquises grâce à I'usage des puerets comme l'implémentation de certaines qualités personnelles, usage également retrouvé chez les souchets maîtres.

Par ailleurs, les techniques de préparation de ces plantes varient, non de manière individuelle mais en fonction du groupe auquel elles appartiennent. Le plus souvent, les aracées d'union sont préparées sous forme de poudre, tout comme les puerets ; les aracées et les souchets de charme sont utilisés sous forme de broyats de plante. Des similitudes d'usage apparaissent nettement entre les aracées d'union et les ptéridophytes ainsi qu'entre les souchets de charme et les aracées de charme. Le groupe des souchets maîtres apparait, telle une catégorie singulière, éventuellement proche des anciens usages des puerets.

Les aracées semblent être des sortes de plantes intermédiaires. Tout d'abord, ces plantes apprécient principalement les sols ensoleillés comme les berges des rivières. Initialement, elles sont sauvages. Afin d'être cultivées, elles sont récoltées à la suite d'un rituel d'enfouissement des cendres d'une proie. Cette forme de paraculture n'a pas été constatée pour d'autres espèces et en ce qui concerne les plantes qui intéressent cet article : les souchets sont domestiqués et les ptéridophytes sont sauvages. Il s'agit là de la principale différence entre ces groupes de plantes. D'un point de vue écosystémique, les aracées se plaisent sur les contreforts amazoniens de basses altitudes. Elles sont absentes dans les zones d'occupation haute des Yanesha. Dès lors, les similarités constatées entre, d'une part, aracées d'union et ptéridophytes, et d'autre part, aracées et souchet de charme font sens et il est possible de postuler que les ptéridophytes seraient nées des aracées d'union et les souchets de charme des aracées de charmes. Dans le souci de faire perdurer un usage, les Yanesha auraient pu le transférer sur un autre groupe de plantes. Le changement de support végétal aurait pu permettre de pallier à une variation partielle d'écologie. Ainsi, les souchets maîtres se seraient multipliés afin de compter parmi eux les souchets de charmes et les ptéridophytes, les fougères d'union. En considérant que l'indication d'usage d'une plante représente la nature de la qualité pour laquelle elle est reconnue et utilisée, l'attribution d'un usage à une plante peut être repensé comme l'attribution d'une vitalité à une autre vitalité végétale tout comme il est possible d'agrémenter de vitalités végétales les vitalités humaines (Valadeau, 2012 : 68). Naît alors ici I'idée de réelles possibilités d'interaction plante-plante.

La question relative à l'origine de la distribution des vitalités des plantes - des usages - dans deux groupes de plantes distinctes au lieu d'un seul se pose également. II est possible de penser que les vitalités des aracées de charme aient trouvé une place au sein des souchets puisque les souchets maîtres permettent

10 À cet égard : hunting magic is quite unlike pushanga, love magic, which is directed at one specific person (Gow, 1991 : 125). 
d'acquérir des capacités spécifiques. Les qualités d'exceller à la chasse comme à la pêche auraient donc pu s'y associer et emprunter ce support végétal. Certaines cypéracées seraient ainsi devenues des souchets de charme. Le mythe narrant l'apparition des souchets maîtres n'a pas été trouvé. Les discours s'accordent autour du fait que Yato' Yos les aurait créés et distribués. Toutefois, une anecdote autour de deux sœurs, aujourd'hui considérées comme les Mères des plantes et dont l'apparence est celle de grands agaves, les donne comme créatrices des souchets par transformation de leurs enfants. Les deux origines différentes des souchets maitres et des souchets de charme laissent penser à une intégration de vitalités de charme au sein des cypéracées.

Pourquoi l'usage des aracées d'union n'aurait-il pas pu être transféré de la même manière vers les souchets ? L'apparition d'un groupe des ptéridophytes semble incertaine. Contrairement aux souchets de charme dont le mythe est semblable à d'autres narrations d'origine plus récente, tel celui de la fièvre jaune, de la variole, de la varicelle et du paludisme et dans lequel un groupe de plantes fut également objet d'échange, le mythe des puerets s'intègre volontiers aux épisodes relatant la spéciation. Cela laisse présupposer que les puerets sont plus anciens que les souchets de charme. Dans ce cas, ce n'est pas le support qui a changé, mais l'usage. Ces habilités pourraient s'être transformées, passant de leurs formes de maîtrise ancienne à d'autres plus récentes, semblables à celles des aracées d'union. Dans ce cas, il est possible de penser à l'arrivée d'un usage sans support provenant des basses terres : lors de leur migration, les Yanesha auraient intégré les aracées d'union des sociétés des terres basses, les exportant ensuite vers les terres montagneuses. Ces aracées ne s'adaptent pas au climat d'altitude et «partent ou retournent sur les berges des fleuves de la vallée » comme le dit Francisco E.11. L'indication d'usage, entendu par leur vitalité, restant sans support aurait pu trouver refuge sur les corps végétaux des puerets expliquant l'évolution de leurs anciens usages vers les actuels.

Ces transpositions ou permutations entre vitalités de plantes - usages - et supports végétaux démontrent cette particularité dynamique des savoirs liés aux plantes et de la flore elle-même. Le caractère miscible des vitalités leur permet de se fondre sur d'autres supports conférant à ceux-ci les qualités qu'elles apportent. Ce nouveau support végétal répond aux exigences principalement définies par la préparation et la forme du remède qui sont conditionnées par les principes de fonctionnalité des corps. Les usages peuvent donc changer de support végétal tout comme les plantes peuvent voir leurs usages se transformer. L'idée de l'élaboration de répertoires de plantes comme outil indispensable à l'ancrage d'un savoir végétal ne peut manifestement pas remplir le rôle escompté. En revanche, c'est la nature du processus conduisant de la quête de la matière végétale utile à l'administration du remède qui semble revêtir une forme plus stable. Ainsi, la connaissance de

11 II s'agit de la réponse à la question posée au sujet de la domestication des aracées en terres hautes. La tentative avait été faite par Francisco E. à partir d'un rhizome offert par un membre de sa famille vivant dans les basses terres. 
I'association d'un usage à une forme végétale n'a pas de stabilité réelle et leurs répertoires suggèrent des enchevêtrements de pharmacopées hybrides.

\section{Referencias citadas}

AIKHENVALD, A. Y., 1999 - The Arawak Language Family. In: The Amazonian languages (M. W. Dixon \& A. Y. Aikhenvald, eds.): 65-105 ; Cambridge: Cambridge University Press.

ALBERT, B., 1985 - Temps du sang, Temps des cendres. Représentation de la maladie, espace politique et système rituel chez les Yanomami du Sud-Est (Amazonie brésilienne), 833 p.; Paris : Université de Paris $X$ - Nanterre. Thèse de doctorat.

ALEXIADES, M. N., 1999 - Ethnobotany of the Ese Eja: Plants, Health and Change in an Amazonian Society, 267 p.; New York City : University of New York. Thèse de doctorat.

BELAUNDE, L. E., 2012 - Diseños Materiales E Inmateriales: La Patrimonialización del Kené Shipibo-Konibo Y De La Ayahuasca En El Perú. Mundo Amazónico, 3 : 123-146.

BELLIER, I., 1986 - La part des femmes : Essai sur les rapports entre les femmes et les hommes Mai Huna (Amazonie Péruvienne), 755 p.; Paris: EHESS. Thèse de doctorat.

BENNETT, B. C., 1989 - Useful Plants of Amazonian Ecuador. Third progress report; USAID: http://pdf.usaid.gov/pdf_docs/PNABM954.pdf

BROWN, M. F., 1984 - The Role of Words in Aguaruna Hunting Magic. American Ethnologist, 11 (3) : 545-558.

BROWN, M. F. \& VAN BOLT, M. L., 1980 - Aguaruna Jivaro Gardening Magic in the Alto Rio Mayo, Peru. Ethnology, 19 (2) : 169-190.

CARID NAVEIRA, M. \& PÉREZ GIL, L., 2002 - Los yaminawa del río Mapuya (Alto Ucayali, Perú). In : Rapport Scientifique Final du Projet TSEMIM, 168 p.; Bruxelles : Commission Européenne CCE_DG XII Recherche, Université Libre de Bruxelles, Centre d'Anthropologie Culturelle.

CARNEIRO, R. L., 1970 - Hunting and Hunting Magic among the Amahuaca of the Peruvian Montaña. Ethnology, 4 (9) : 331-341.

CHAPUIS, J., 1998 - La personne Wayana entre sang et ciel. Parties 2 et 3, 1284 p.; AixMarseille : Université d'Aix-Marseille. Thèse de doctorat.

CHAUMEIL, J.-P., 1993 - Voir, savoir, pouvoir. Le chamanisme chez les Yagua du Nord-Est péruvien, 349 p.; Paris : Geord Ed., Col. Ethnos.

DEL AGUILA, J. \& DÉLÉAGE, P., 2010 - Mythologie Sharanahua Traduction provisoire : Narrations (2001-2004) Barisuhui, Fahuido, Fasharoadi, Inahuapidi, Ishaba, Taradi, Yabafacu, Yabayahua, $74 \mathrm{p}$.

DÉLÉAGE, P., 2005 - Le chamanisme sharanahua. Enquête sur l'apprentissage et l'épistémologie d'un rituel, 418 p.; Paris : EHESS. Thèse de doctorat.

FOLLIARD, T., 2008 - Phytothérapie externe sur le continent américain - Amazonie et Amérique du Nord (partie 2). Phytothérapie, 6 : 232-246.

GOW, P., 1991 - Of mixed blood. Kinship and history in Peruvian Amazonia, 331 p.; Oxford: Clarendon Press. 
GRENAND, P., 1980 - Introduction à l'étude de l'univers Wayapi, Ethnoécologie des Indiens du Haut-Oyapock (Guyane Française), 332 p.; Paris : SELAF.

GRENAND, F., 2001 - Stratégies de nomination des plantes cultivées dans une société tupiguarani, les Wayãpi. AMERINDIA, 26/27 : 1-36.

GRENAND, P. \& FLEURY, M., 2012 - Le voyage discret des plantes : Abelmoschus moschatus (Malvaceae) et Zingiber zerumbet (Zingiberaceae) en Amérique tropicale. Revue d'ethnoécologie, 1 : 1-19.

GUTIERREZ CHOQUEVILCA, A. L., 2013 - Face-à-face interspécifiques et pièges à pensée des Quechua de Haute Amazonie (Pastaza). L'Herne, 9 : 33-47.

HECKLER, S. L., 2004 - Cultivating Sociality: Aesthetic Factors In The Composition And Function Of Piaroa Home gardens. Journal of Ethnobiology, 24 (2) : 203-232.

JAUREGUI, X., CLAVO, Z.M., JOVEL, E.M. \& PARDO-DE-SANTAYANA, M., 2011 - “Plantas Con Madre": Plants That Teach And Guide In The Shamanic Initiation Process In The East-Central Peruvian Amazon. Journal of Ethnopharmacology, 134 : 739-752.

KATZ, E., LOPEZ, C. L., FLEURY, M., MILLER, R. P., PAYÊ, V., DIAS, T., SILVA, F., OLIVEIRA, Z. \& MOREIRA, E., 2012 - No Greens In The Forest? Note On The Limited Consumption Of Greens In The Amazon. Acta Societatis Botanicorum Poloniae, 81 (4) : 283-293.

KAWA, N. C., 2011 - The Social Nature Of Agrobiodiversity In Central Amazonia, 203 p.; Florida: University of Florida. Thèse de doctorat.

KOHN, O. E., 2002 - Natural Engagements And Ecological Aesthetics Among The Ávila Runa Of Amazonian Ecuador, 495 p.; Wisconsin : University Of WisconsinMadison. Thèse de doctorat.

LELONG, B., 1974 - La rivière du Maïs, 226 p.; Paris : Lattés.

LENAERTS, M., 2002 - Nos sœurs Manioc et l'étranger Jaguar : diversité et changement des savoirs sur les Êtres de la Nature chez les Ashéninka de l'Ucayali, 450 p. ; Bruxelles : Université Libre de Bruxelles. Thèse de doctorat.

LUZIATELLI, G., SØRENSEN, M., THEILADE, I. \& MØLGAARD, P., 2010 - Asháninka Medicinal Plants: a Case Study from the Native Community of Bajo Quimiriki, Junín, Peru. Journal of Ethnobiology and Ethnomedicine, 6 (21) : 1-23.

MADISON, M., 1981 - Notes on caladium (araceae) and its allies. Selbyana, 5 (3/4) : 342-377.

MAXWELL, N., 1970 - Actitudes de cuatro tribus de la selva peruana respecto a plantas empleadas como anticonceptivos por vía oral. In: Actas y Memorias vol. 4: 101107; Lima: XXXIX Congreso Internacional de Americanistas.

MILLIKEN, W. \& ALBERT, B., 1996 - The Use of Medicinal Plants by the Yanomami Indians of Brazil. Economic Botany, 50 (1) : 10-25.

OCHOA-GILONNE, N., 2007 - Entre plusieurs mondes. Les chayahuita de I'Amazonie péruvienne, 355 p.; Paris : EHESS. Thèse de doctorat.

ODONNE, G., BOURDY, G., OCHOA SIGUAS, N. \& ALBAN-CASTILLO, J., 2014 - Plantas medicinales y de otros usos de los Shawis, 414 p.; Lima : CONCYTEC, FONDECYT, IRD.

ODONNE, G., VALADEAU, C., ALBAN-CASTILlO, J., STIEN, D., SAUVAiN, M. \& BOURDY, G., 2013 - Medical ethnobotany of the Chayahuita of the Paranapura basin (Peruvian Amazon). Journal of Ethnopharmacology, 146 (1) : 127-131.

RAMA-LECLERC, F., 2003 - Des modes de socialisation par les plantes chez les ShipiboConibo d'Amazonie péruvienne, une étude des relations entre humains et nonhumains dans la construction sociale ; Paris : Université Paris X - Nanterre. Thèse de Doctorat. 
RIVAS RUIZ, R., 2004 - La pesca en dos pueblos de ríos andino-amazónicos: Ashaninka y Yanesha. In: Las nociones del mal y el temor en las cosmovisiones aborígenes de América (M. Califano, M. C. Dasso \& E. Fernández, eds.): 119-184 ; Buenos Aires : CIAFIC Ed.

SANTOS-GRANERO, F., 1998 - Writing History Into the Landscape: space, myth, and ritual in Contemporary Amazonia. American Ethnologist, 25 (2) : 128-148.

SCHULTES, R. E. \& HOFMAN, A., 1979 - Les plantes des dieux, 208 p.; Paris : Berger Levrault.

SHEPARD, G. H., 1999 - Pharmacognosy and the Senses in Two Amazonian Societies ; Berkeley: University of California. Thèse de doctorat.

SMITH, R. C., 1999 - Caciques chinchaycochas, funcionarios incas y sacerdotes amueshas: los caminos antiguos de Chinchaycocha hacia la selva central. Cultura andina, (3) : 59-71.

TOURNON, J. \& SILVA, M., 1988 - Plantas para cambiar el comportamiento humano entre los Shipibo-Conibo. Antropologica, 6 (6) : 161-176.

TOURNON, J., RAYNAL-ROQUES, A. \& ZAMBETTAKIS, C., 1986 - Les Cypéracées médicinales et magiques de I'Ucayali. Journal d'agriculture traditionnelle et de botanique appliquée, $33:$ 213-224.

VALADEAU, C., 2012 - Médecine chez les yanesha d'Amazonie péruvienne - La traversée par les plantes, 383 p. ; Paris : L'harmattan.

VAN ANDEL, T., RUSSCHAERT, S., BOVEN, K. \& DALY, L., 2015 - The use of Amerindian charm plants in the Guianas. Journal of Ethnobiology And Ethnomedicine, $11: 66$.

ZENT, S. \& ZENT, E., 2012 - Jodï horticultural belief, knowledge and practice: incipient or integral cultivation? Boletim do Museu Paraense Emílio Goeldi. Ciências Humanas, 7 (2) : 293-338. 